\title{
On the Weights of Nations: Assigning Voting Weights in a Heterogeneous Union
}

\author{
Salvador Barberà \\ Universitat Autonoma de Barcelona and Spanish Ministry of Education and Science \\ Matthew O. Jackson \\ California Institute of Technology
}

\begin{abstract}
We study indirect democracy in which countries, states, or districts each elect a representative who later votes at a union level on their behalf. We show that the voting rule that maximizes the total expected utility of all agents in the union involves assigning a weight to each district's vote and then sticking with the status quo unless at least a threshold of weighted votes is cast for change. We analyze how the weights relate to the population size of a country and the correlation structure of agents' preferences, and then we compare the voting weights in the Council of the European Union under the Nice Treaty and the recently proposed Constitution.
\end{abstract}

\section{Introduction}

Citizens vote occasionally, whereas their elected representatives vote frequently. This is sensible because of the burden of becoming informed

We gratefully acknowledge financial support from the Guggenheim Foundation, the Center for Advanced Studies in the Behavioral Sciences, and the National Science Foundation under grants SES-9986190 and SES-0316493, as well as financial support from the Centre de Referència d'Economia Analítica (Barcelona), the Spanish Ministry of Science and Technology through grant BEC2002-002130, and the Generalitat of Catalonia through grant SGR2001-00162. We thank Claus Beisbart, Ken Binmore, Jon Eguia, Annick Laruelle, Giovanni Maggi, Vincent Merlin, and Federico Valenciano for helpful discussions and comments, and Robert Shimer and two referees for suggestions on earlier drafts. We are also very grateful to Danilo Coelho for research assistance.

[Journal of Political Economy, 2006, vol. 114, no. 2]

(C) 2006 by The University of Chicago. All rights reserved. 0022-3808/2006/11402-0005 $\$ 10.00$ 
on a myriad of issues and the cost of involving full populations in all the decisions that direct democracy would require. While indirect democracy is sensible and prevalent, it introduces distortions in the decision process because a single vote by a representative does not completely represent the heterogeneity of votes that would be cast by that representative's constituency.

If districts are small, of similar size, and of similar degrees of heterogeneity, then weighting each representative's vote equally provides a system of indirect democracy that maximizes overall societal welfare. However, for a variety of reasons, there are many systems of indirect democracy that are not structured in this way. A particularly important and timely example is the Council of Ministers of the European Union, a critical decision-making body of the European Union. That council consists of a single representative from each country in the European Union. The countries differ widely in their population sizes and compositions. Similar examples include the United Nations, the U.S. Senate, and a variety of state and local governments. In any democratic union in which the districts are of different sizes and compositions, it makes sense to weight the votes of the representatives. ${ }^{1}$ For instance, if districts differ in population and votes are not weighted, then small districts could impose decisions that a majority of citizens oppose.

In this paper, we take as given that a heterogeneous set of countries, states, or districts each have one representative who votes on their behalf over collective decisions. We characterize the voting rule that maximizes total societal welfare, as measured by the sum of the utilities of all citizens of the union, subject to the constraint that the district structure is fixed exogenously and is possibly heterogeneous. We examine votes over two alternatives: a status quo and change. We show that an optimal voting rule consists of a weight for each country's vote and a threshold, indicating how large the total weight of votes cast in favor of change must be in order for change to be enacted.

One important conclusion of our analysis is that the optimal voting weights and thresholds can be derived separately. The optimal weight of a country's vote depends on the size of the population and the distribution of preferences within a country relative to other countries. The threshold depends on the bias of preferences in terms of the intensity in favor of the status quo compared to change.

The efficient weights can be described intuitively as follows. Consider the vote by a given representative of a country. Suppose that he or she has voted yes on a given issue. We can then ask the following question: Given the vote of yes, what is the surplus of people in the country who

\footnotetext{
${ }^{1}$ Alternatively, one can think of adjusting the number of representatives that each country, state, or district has.
} 
favor yes over no? For instance, if 62 percent of the people favor yes and 38 percent favor no, then 24 percent more of the population favor yes versus no. Multiplying this percentage times the population gives us a measure of how much this country would benefit if we chose yes versus no and how much this country would suffer if we chose the reverse. The efficient voting weight is exactly this expected surplus. ${ }^{2}$

As the general characterization of efficient voting rules depends on the distribution of preferences within each country, we also explore a model that we refer to as the "block model," which allows us to derive optimal weights as a function of population size. This works by assuming that a country's population can be partitioned into blocks: citizens within a block have perfectly correlated preferences, whereas citizens across blocks have independent preferences. This structure allows us to pinpoint the efficient voting weights and thresholds under two focal scenarios.

We also examine the model's implications for the voting system of the Council of Ministers of the European Union. The Nice Treaty of 2000 and the Constitutional Convention of 2003 proposed different sets of weights and different voting thresholds. Under the Nice Treaty, weights are less than proportional to population size and the threshold is relatively high (73.9 percent). The Constitutional Convention proposed weights that are directly proportional to population size and a lower threshold (65 percent) ${ }^{3}$ We show that these two conflicting proposals coincide with the optimal weights under two polar cases of our "block model." Which weights are more efficient then boils down to an empirical question of preference patterns.

Contribution and relation to the literature.-It is surprising that the previous literature has not considered the criterion of efficiency (total expected utility) as a guide to determine optimal voting rules for indirect democracy. ${ }^{4}$ The literature on indirect democracy approaches the problem from other perspectives. For instance, there is a rich literature in

\footnotetext{
${ }^{2}$ Our model allows for heterogeneities in intensities of preferences among voters as well, and the weights adjust for that. Here we are simply describing a special case in which intensity among voters is similar. The full characterization is provided below.

${ }^{3}$ The convention's proposal also includes a requirement that 55 percent of the countries support a measure (Constitution, Title IV, Article I-25), which could also be binding, but less frequently; and that a blocking minority include at least four countries. (There are also special provisions for votes on issues that were not proposed through either the commission or the Union Minister for Foreign Affairs, where the requirement on countries is raised to 72 percent.) As a first (rough) approximation, we ignore these extra constraints in the discussion of the Constitution's proposed weights. As we shall see in the discussion following theorem 1, more complex voting systems can be optimal (see also Harstad [2005] for a rationalization of dual majority systems).

${ }^{4}$ Rae (1969) analyzed voting rules under this utilitarian perspective of maximizing expected utility or satisfaction rather than decisiveness (see also Badger 1972; Curtis 1972), but in the context of direct democracy.
} 
cooperative game theory on weighted majority games. A main thread there has been to produce power indices, such as the Shapley-Shubik (1954) and Banzhaf (1965) indices, which measure things such as the relative probabilities that different voters are pivotal. While some researchers have built power measures based on satisfaction (i.e., total utility) and contrasted them with power measures built on decisiveness (see, e.g., Dubey and Shapley 1979; Barry 1980; Laruelle and Valenciano 2003), our perspective is still quite different. Our aim is not to measure power or satisfaction or to compare rules under such measures, but instead to study the optimal design of voting rules. To the extent that the previous literature has thought about designing rules, it has focused on equating the power of agents rather than maximizing the total expected utilities of agents. ${ }^{5}$ This dates to the seminal work of Penrose (1946). These two objectives can lead to quite different voting rules, and, as we show, maximizing total expected utility can result in large inequalities in the treatment of individuals across countries.

Perhaps the closest predecessor to the theoretical part of our work is the article by Felsenthal and Machover (1999), who also study the design of two-stage voting rules from an optimization perspective. Their objective is to minimize the expected difference between the size of the majority and the number of supporters of the chosen alternative. ${ }^{6}$ That objective differs from maximizing total expected utility since it does not account for the surplus of voters in favor of an alternative when the majoritarian alternative is selected, but accounts for the deficit only when the majoritarian alternative is not selected. ${ }^{7}$

Finally, researchers have also examined the European Union's decision making and brought ideas from weighted games to assess the relative power of different countries under the Nice Treaty (see, e.g., Laruelle [1998], Laruelle and Widgrén [1998], Sutter [2000], Baldwin et al. [2001], Bräuninger and König [2001], Galloway [2001], and Leech [2002] and some of the references cited there). As the foundations of our analysis of voting rules differ from the previous literature, so does our analysis of the Nice Treaty and the new Constitution. Among other things, we identify correlation structures of citizens' preferences that

\footnotetext{
${ }^{5}$ There are exceptions in the recent literature (e.g., Aghion and Bolton 2003; Barberà and Jackson 2004a; Harstad 2004; Casella 2005), but they approach the problem from very different perspectives.

${ }^{6}$ Felsenthal and Machover's article includes an illuminating discussion of their objective and some of the imprecisions in the previous literature.

${ }^{7}$ While these two perspectives differ, they lead to the same weights in the particular case of large countries of independently and identically distributed voters, where the weights are proportional to the square root of a country's population size (as originally suggested by Penrose [1946] from an even different perspective). The setting with a large number of independently and identically distributed voters is special and not so realisticespecially for applications to, e.g., the European Union.
} 
would justify the various rules that have been proposed, something that has not appeared previously.

Since writing this, we have become aware of independent work by Bovens and Hartmann (2004), Feix et al. (2004), and Beisbart, Bovens, and Hartmann (2005), who examine efficiency as an issue in the definition of a voting rule. However, the works are (completely) complementary. ${ }^{8}$

\section{An Example}

A simple example gives a preview of some of the issues that arise in designing an efficient voting rule. The example shows why in some cases it will be efficient to use weights that are not proportional to population.

EXAmple 1. Nonproportional versus Proportional Weights

Consider a world with three countries. Countries 1 and 2 have populations of one agent each. Country 3 has a population of three agents. Each agent has an equal probability of supporting alternative $a$ and alternative $b$, and preferences are independent across agents. Agents get a payoff of one if their preferred alternative is chosen and minus one if the other alternative is chosen. Thus total utility can be deduced by keeping track of the number of agents who support each alternative.

First, consider a situation in which countries are weighted in proportion to their populations and use a threshold of 50 percent of the total weight. That would result in weights of $(1,1,3)$ and a threshold of 2.5. This is equivalent to letting country 3 choose the alternative.

Note that it is possible for a minority of agents to prefer an alternative and still have that be the outcome. For instance, if two agents in country 3 prefer $a$ and all other agents prefer $b$, then $a$ is still chosen.

The "efficient" weights-that is, those that maximize the total expected utility-are $(1,1,1.5)$, and the efficient threshold is 1.75 . In this situation, the efficient voting rule is thus equivalent to one vote per country. The proof that this is the efficient rule comes from our characterization theorem below. However, to get a feeling for why it differs from the straight proportional rule, let us compare the expected utilities.

Under the efficient rule it is also still possible for a minority of agents to prefer $a$ and a majority to prefer $b$ but to still have $a$ selected. For instance, this happens if agents in countries 1 and 2 prefer $a$ but all

\footnotetext{
${ }^{8}$ Our characterization results, block models, and examination of the E.U. data differ from their analyses. Bovens and Hartmann examine combinations of maximin and utilitarian (efficient) measures and examine when degressive proportionality is justified, and Beisbart et al. provide a Pareto ranking of several variations on potential rules for the E.U. Council of Ministers under various preference configurations. Feix et al. contrast two models of voter preferences-impartial culture and impartial anonymous culture-and show through simulations that they can lead to different optimal voting rules.
} 
agents in country 3 prefer $b$. Despite the fact that the efficient voting rule is not always making the correct choice in terms of maximizing the total utility, there is an important distinction between the efficient rule and the proportional rule here. Fewer configurations of preferences under the efficient voting rule lead to incorrect (minority-preferred) decisions than under the proportional voting rule. This is seen as follows.

The configurations that are problematic in terms of agents' preferences are as follows (where the first two entries indicate the preferences of agents in the first two countries, and the last three entries correspond to the agents in country 3): Alternative $a$ can be the outcome and be preferred only by a minority under the efficient voting rule only when preferences are $(a ; a ; b, b, b)$. However, under the proportional voting rule there are three preference configurations that can lead to the choice of $a$ when a majority prefers $b$. These are $(b ; b ; a, a, b),(b ; b ; a$, $b, a)$, and $(b ; b ; b, a, a)$.

When we compute the total expected utility (summed across all agents), it is 1.75 under the efficient voting rule compared to 1.5 under the proportional voting rule.

\section{The Model}

\section{A. Decisions and Agents}

A population of agents is divided into $m$ countries. Country $i$ consists of $n_{i}$ agents, and this set is denoted by $C_{i}$. The total number of agents in the union is $n=\sum_{i} n_{i}$.

The agents make a decision between two alternatives labeled $a$ and $b$. We refer to $b$ as the status quo and $a$ as change.

A state of the world is a description of all agents' preferences over the two alternatives. Given that there are only two alternatives, we need only keep track of the difference in utility that an agent has for alternatives $a$ and $b$. Therefore, without loss of generality we normalize things so that agent $j$ gets a utility of $u_{j}$ if $a$ is chosen and a utility of zero if $b$ is chosen. So if $u_{j}$ is positive, then $j$ prefers $a$.

A state of the world is thus a vector $\boldsymbol{u} \in \mathbb{R}^{n}$, with element $u_{j}$ being the difference between agent $j$ 's utilities for $a$ and $b$. The uncertainty regarding the state is described by a probability distribution, and all expectations are taken with respect to that distribution.

\section{B. A Two-Stage Voting Procedure}

The decision-making process is described as follows. 
The First Stage

In the first stage, $\boldsymbol{u}$ is realized and each country's representative decides whether to vote for $a$ or vote for $b$. Generally, the representative's vote will be related to the preferences of the agents in the representative's country.

The representative's voting behavior is represented by a function $r_{i}: \mathbb{R}^{n} \rightarrow\{a, b\}$, which maps the state into a vote. The notation $r_{i}(\boldsymbol{u})=$ $a$ indicates that the representative of country $i$ votes for $a$ and $r_{i}(\boldsymbol{u})=$ $b$ indicates that the representative votes for $b$, given the state $\boldsymbol{u}$. We assume that $r_{i}$ depends only on the preferences of agents within country $i$.

It is important to emphasize that this formulation allows for many different ways in which the representative's vote could depend on the state of agents' preferences. It is conceivable that the representative is an existing politician who polls the population or that the representative is a dictator, bureaucrat, or other who might decide how to vote quite differently. Later in the paper we consider the prominent case in which the representative votes in accordance with a majority of the population.

\section{The Second Stage}

In the second stage, the votes of the representatives are aggregated according to a voting rule.

Let $v: \mathbb{R}^{n} \rightarrow\left\{0, \frac{1}{2}, 1\right\}$ denote the outcome of this two-stage voting procedure as a function of the state. Here $v(\boldsymbol{u})=1$ is interpreted as meaning that alternative $a$ is chosen, $v(\boldsymbol{u})=0$ means that alternative $b$ is chosen, and $v(\boldsymbol{u})=\frac{1}{2}$ denotes that a tie has occurred and a coin is flipped.

Feasible voting rules are those that depend only on the information obtained through the votes of the representatives. The set of all feasible voting rules thus are those in which $v(\boldsymbol{u}) \neq v\left(\boldsymbol{u}^{\prime}\right)$ implies that $r_{i}(\boldsymbol{u}) \neq$ $r_{i}\left(\boldsymbol{u}^{\prime}\right)$ for at least one country $i$.

Given this coding of $v(\boldsymbol{u})$, the utility of agent $j$ in state $\boldsymbol{u}$ can now be written as $v(\boldsymbol{u}) \times u_{j}$. Thus the total utility summed across all agents in the union is

$$
v(\boldsymbol{u}) \sum_{j} u_{j}
$$

and the total expected utility of a union using a voting rule $v$ is

$$
E\left[\sum_{j} v(\boldsymbol{u}) u_{j}\right]
$$




\section{Weighted Voting Rules}

An important subclass of feasible voting rules are weighted voting rules. In such rules, the vote of the representative of country $i$ is given a weight

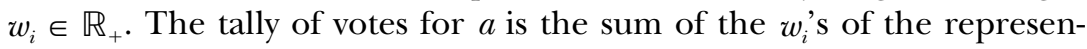
tatives who cast votes for $a$, and similarly for $b$. Alternative $a$ is selected if its tally of weights exceeds the threshold (denoted $\tau \in\left[\begin{array}{ll}0, & \Sigma_{i} w_{i}\end{array}\right]$ ), alternative $b$ is selected if the tally of weights for $a$ is less than the threshold, and ties are broken by the flip of a fair coin.

\section{Equivalent Voting Rules}

When one is considering weighted voting rules, different weights and thresholds can lead to the same voting rule, and so weighted voting rules are defined only up to an equivalence class. ${ }^{9}$ Instead of defining two different pairs of weights and thresholds to be equivalent if their induced voting rules always make the same choices, we need a coarser requirement for our main results because tie breaking is not completely tied down under efficient voting rules.

We say that a profile of voting weights and a threshold $(w, \tau)$ with induced weighted voting rule $v$ is equivalent up to ties to a profile of voting weights and a threshold $\left(w^{\prime}, \tau^{\prime}\right)$ with induced weighted voting rule $v^{\prime}$ if $v(\boldsymbol{u})=v^{\prime}(\boldsymbol{u})$ for all $\boldsymbol{u}$ such that $v^{\prime}(\boldsymbol{u}) \neq \frac{1}{2}$.

This is not quite an equivalence relationship, since it allows $v$ to break ties differently from $v^{\prime} \cdot{ }^{10}$ To see why we define equivalence only up to ties, consider a simple example. There are two countries, and each consists of a single agent whose utilities take on values in $\{-1,1\}$. Let $w^{\prime}$ be $(1,1)$ and the threshold be 1 . Note that the induced voting rule $v^{\prime}$ would be efficient for this example. When things are unanimous, $v^{\prime}$ picks the unanimous choice; but when $u_{1}$ and $u_{2}$ have opposite signs, the rule flips a coin and so $v^{\prime}(\boldsymbol{u})=\frac{1}{2}$. Alternative weights $w=(1+\epsilon$, 1 ) with a threshold of $1+(\epsilon / 2)$ would also be efficient but would favor the first agent in the case of a tie. Thus the induced voting rule $v$ would be more resolute than $v^{\prime}$ but would make the same choices in any case in which efficiency was at stake.

\footnotetext{
${ }^{9}$ Equivalent voting rules can be rescalings of each other but also might not be. For instance, with three countries, $w=(3,2,2)$ with a threshold of 3.5 is equivalent to $w^{\prime}=(1,1,1)$ with a threshold of 1.5 : they both select the alternative that at least two countries voted for.

${ }^{10}$ This is an asymmetric relationship: $v$ can be equivalent up to ties with $v^{\prime}$ whereas the reverse might not hold.
} 


\section{Efficient Voting Rules}

Consider the problem maximizing the expected sum of the utilities of all agents in the union. The optimum would be to choose $a$ when $\sum_{j} u_{j}>0$ and $b$ when $\sum_{j} u_{j}<0$. This optimum will generally not be realized, since we lose information in a two-stage procedure. In the second stage we see only the votes of the representatives, which include only indirect information about the preferences of the agents.

Efficient voting rules.-While we cannot always maximize the realized sum of utilities, we can still ask which voting rule maximizes the expected total utility. An efficient voting rule $v$ is one that has a maximal value of

$$
E\left[\sum_{j} v(\boldsymbol{u}) u_{j}\right]
$$

across all feasible voting rules.

\section{A. A Full Characterization of Efficient Voting Rules}

Efficient voting rules work as follows. For each country assign two weights: one for $a$ votes and one for $b$ votes. ${ }^{11}$ Country $i$ 's weight for $a$ votes is

$$
w_{i}^{a}=E\left[\sum_{k \in C_{i}} u_{k} \mid r_{i}(\boldsymbol{u})=a\right]
$$

and its weight for $b$ votes is

$$
w_{i}^{b}=-E\left[\sum_{k \in C_{i}} u_{k} \mid r_{i}(\boldsymbol{u})=b\right] .
$$

The efficient voting rule $v^{E}(\boldsymbol{u})$ is then defined by

$$
v^{E}(\boldsymbol{u})= \begin{cases}1 & \text { if } \sum_{i: r_{i}(\boldsymbol{u})=a} w_{i}^{a}>\sum_{i: r_{i}(\boldsymbol{u})=b} w_{i}^{b} \\ 0 & \text { if } \sum_{i: r_{i}(\boldsymbol{u})=a} w_{i}^{a}<\sum_{i: r_{i}(\boldsymbol{u})=b} w_{i}^{b} \\ \frac{1}{2} & \text { if } \sum_{i: r_{i}(\boldsymbol{u})=a} w_{i}^{a}=\sum_{i: r_{i}(\boldsymbol{u})=b} w_{i}^{b} .\end{cases}
$$

Theorem 1. If preferences are independent across countries, then a voting rule is efficient if and only if it is equivalent up to ties to $v^{E}$.

\footnotetext{
${ }^{11}$ This is a feasible voting rule but not a weighted voting rule.
} 
The proof appears in the Appendix.

The intuition behind the theorem is straightforward. Conditional on a vote of $r_{i}(\boldsymbol{u})=a, w_{i}^{a}=E\left[\sum_{k \in C_{i}} u_{k} \mid r_{i}(\boldsymbol{u})=a\right]$ is the estimate of the total utility support for $a$ in country $i$. By weighting a country's votes in proportion to these expectations, the voting rule chooses the alternative that will result in the highest total utility based on what can be inferred from the votes of the representatives.

To get a feeling for how such rules work, consider an example with three countries, where $1=w_{1}^{a}=w_{1}^{b}=w_{2}^{a}=w_{2}^{b}=w_{3}^{a}$ but $w_{3}^{b}>2$. Here all votes are equally informative, except when country 3 votes for $b$, which indicates stronger support for $b .^{12}$ In this case, the efficient rule is to choose $b$ whenever country 3 votes for $b$ and otherwise to operate under majority rule. ${ }^{13}$ Note that this rule cannot be represented as an ordinary weighted voting rule in which each country is just given some weight and there is some threshold needed for change. There is an asymmetry between how country 3's vote for $b$ is treated compared to all other votes.

Before we turn to the application to the European Union, let us discuss a few of the implications of the formula. The assumption of the independence of voters' preferences across countries is restrictive and is important to the conclusions of the theorem. Without this assumption, the estimation of one country's utility for a given alternative would depend on the full profile of votes of all countries. In that case an optimal voting rule would no longer be a weighted rule, but a rule that was a much more complex mapping between vectors of votes and decisions, since each country's vote would convey information about the preferences of all countries' electorates.

\section{B. Bias and Weighted Voting Rules}

In many contexts, there might be some asymmetry in agents' preferences over alternatives.

We say that country $i$ is biased with bias $\gamma_{i}>0$ if

$$
E\left[\sum_{k \in C_{i}} u_{k} \mid r_{i}(\boldsymbol{u})=b\right]=-\gamma_{i} E\left[\sum_{k \in C_{i}} u_{k} \mid r_{i}(\boldsymbol{u})=a\right] .
$$

A country's bias captures the difference in expectations concerning how much the country's voters prefer $a$ over $b$ when their representative

\footnotetext{
${ }^{12}$ This could be due to asymmetries in intensities of preferences within country 3 or could be due to the correlation structure of preferences within country 3 .

${ }^{13}$ This is reminiscent of features such as in the U.N. Security Council voting rule, where core countries hold a veto.
} 
votes for $a$, compared to our expectations about how much the country's voters prefer $b$ over $a$ when their representative votes for $b$.

In cases in which there is a common bias factor $\gamma$ across countries, theorem 1 has the following corollary. In that case, $w_{i}^{b}=\gamma w_{i}^{a}$, and the efficient voting rule can be written as a weighted voting rule.

Corollary 1. If preferences are independent across countries and each country has the same bias factor $\gamma$, then a voting rule is efficient if and only if it is equivalent up to ties to a weighted voting rule with weights,

$$
w_{i}^{*}=E\left[\sum_{k \in C_{i}} u_{k} \mid r_{i}(\boldsymbol{u})=a\right]
$$

and a threshold of $\left(\gamma \sum_{i} w_{i}^{*}\right) /(\gamma+1)$.

A prominent case of interest is one in which countries are unbiased $(\gamma=1)$. Then a voting rule is efficient if and only if it is equivalent up to ties to the weights $w_{i}^{*}$ given above and the 50 percent threshold of $\left(\sum_{i} w_{i}^{*}\right) / 2$.

We make several remarks about corollary 1 .

The threshold depends on the bias $\gamma$, whereas the weights are determined by the expectations that come from each country. Thus one can judge whether a voting rule's weights are optimal independently of the threshold, and vice versa.

The extent to which a country's representative's vote is tied to the utilities of the agents in the country has important consequences. For example, a large country with a representative who is a dictator whose vote is uncorrelated with his population's preferences receives a smaller weight than a smaller country with a representative whose vote is very responsive to his population's preferences. More generally, the larger support (in net utility) for an alternative that one infers on the basis of a representative's vote for that alternative, the larger the weight that a country receives.

The weights are affected by the distribution of opinions inside a country. For instance, if a country's agents had perfectly correlated opinions (and the representative voted in accordance with them), then a vote for an alternative would indicate a strong surplus of utility in favor of that alternative. The more independent the population's opinions, the lower the expected surplus of utility in any given situation. Thus higher correlation among agents' utilities will generally lead to higher weights.

The efficient weights take into account the intensity of preferences. So, relatively larger utilities lead to relatively larger weights. Thus a country that cares more intensely about issues is weighted more heavily than a country that cares less, all else held equal. Owing to practical and philosophical difficulties with the appraisals of utilities, one might 


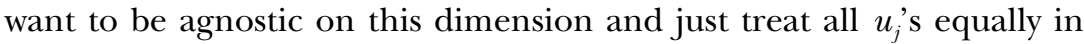
the sense of assigning them only values of plus one or minus one. We do this in the following section.

The following example illustrates the relation between bias and the voting threshold, as well as the separability of weights and thresholds.

EXAMPLE 2. Bias and Thresholds

Consider three countries. Countries 1 and 2 have one voter each. Country 3 has $n_{3}$ voters.

Voters' preferences are biased, with bias factor $\gamma$. All voters are equally likely to prefer $a$ or $b$ and have $u_{j}^{\prime}$ s take on values of either one or $-\gamma$ with equal probability. Thus, when a voter prefers $b$, he or she cares more intensely than when he or she prefers $a$, by a factor $\gamma$. Country 3 's voters have perfectly correlated preferences so that either they all prefer $a$ or they all prefer $b$. So country 3 is just a scaled-up version of countries 1 and 2 .

Theorem 1 tells us that the vector of voting weights should be $(1,1$, $\left.n_{3}\right)$, and the voting threshold should be a fraction of $\gamma /(\gamma+1)$ of the total weight. As $\gamma$ becomes large, unanimity for $a$ is required to overturn the status quo $b$. If $\gamma=1$, then the threshold is 50 percent of the weighted votes.

As we vary $n_{3}$ and $\gamma$, the efficient voting rule takes some interesting forms. For instance, suppose that $n_{3}=3$. Then country 3 has three times as many voters and is relatively favored in terms of weights. However, 3's "power" still depends on the voting threshold. If $\gamma=1$, then the threshold is 50 percent of the weighted votes; then country 3 is the only country that has a nontrivial vote and dictates the choice. However, if $\gamma=2$, then the threshold is two-thirds of the voting weights. Then $a$ passes if and only if country 3 and at least one of 1 or 2 votes for $a$. Either 3 or 1 and 2 together can block $a$ and keep the status quo.

This example shows the separability of how the weights and thresholds are determined. The weights depend on the relative utilities (and thus populations) represented by each of the countries, whereas the threshold depends on the underlying preference structure in terms of a bias for change versus the status quo. It also shows that the overall voting rule can still depend in subtle ways on both the threshold and weights.

\section{A Block Model}

In order to apply the theory and calculate weights as a function of a country's population, we now introduce a model that is more specific about the distribution of agents' preferences and how representatives vote. We call this stylized model the "block model," and it works as follows.

First, we treat agents' utilities equally, in the sense that we only account 
for them as plus one or minus one and disregard personal intensities. This may be defended on grounds of practicality, but also more philosophically as an equal-treatment condition. We also examine a case in which each agent has an equal probability of supporting either alternative.

Second, we assume that representatives vote for the alternative that has majority support in their country, flipping a fair coin when indifferent.

Third, the utilities of agents are distributed as follows. Each country is made up of some number of blocks of agents, where agents within each block have perfectly correlated preferences and preferences across blocks are independent. The blocks within a country are of equal size.

These assumptions reflect the fact that countries are often made up of some variety of constituencies, within which agents tend to have correlated preferences. For instance, the farmers in a country might have similar opinions on a wide variety of issues, as will union members, intellectuals, and so forth. The block model is a stylized but useful way to introduce correlation among voters' preferences, and simple variations of it provide interesting and pointed specifications of optimal voting rules.

\section{A. Efficient Weights in the Block Model}

Let $N_{i}$ be the number of blocks in country $i$. Letting $p_{i}$ be the size of each block, we obtain the following expression for the efficient weight of country $i$ :

$$
w_{i}=p_{i} 2^{-N_{i}} \sum_{x>N_{i} / 2}\left(2 x-N_{i}\right) \frac{N_{i} !}{x !\left(N_{i}-x\right) !} .
$$

There are two prominent variations on the block model that we focus on in what follows. We call the first variation the fixed-size-block model. In this variation, blocks have a fixed size across all countries. In this case, a country's population can be measured in blocks, and a larger country has more blocks than a smaller one. Here the $p_{i}$ 's are the same across all countries.

We call the second variation the fixed-number-of-blocks model. In this variation, all countries have the same number of blocks, and the size of the blocks in a given country adjusts according the country's population size. Here the $N_{i}$ 's are the same across all countries.

We obtain the following expressions for the efficient weights in the two specializations of the block model. 


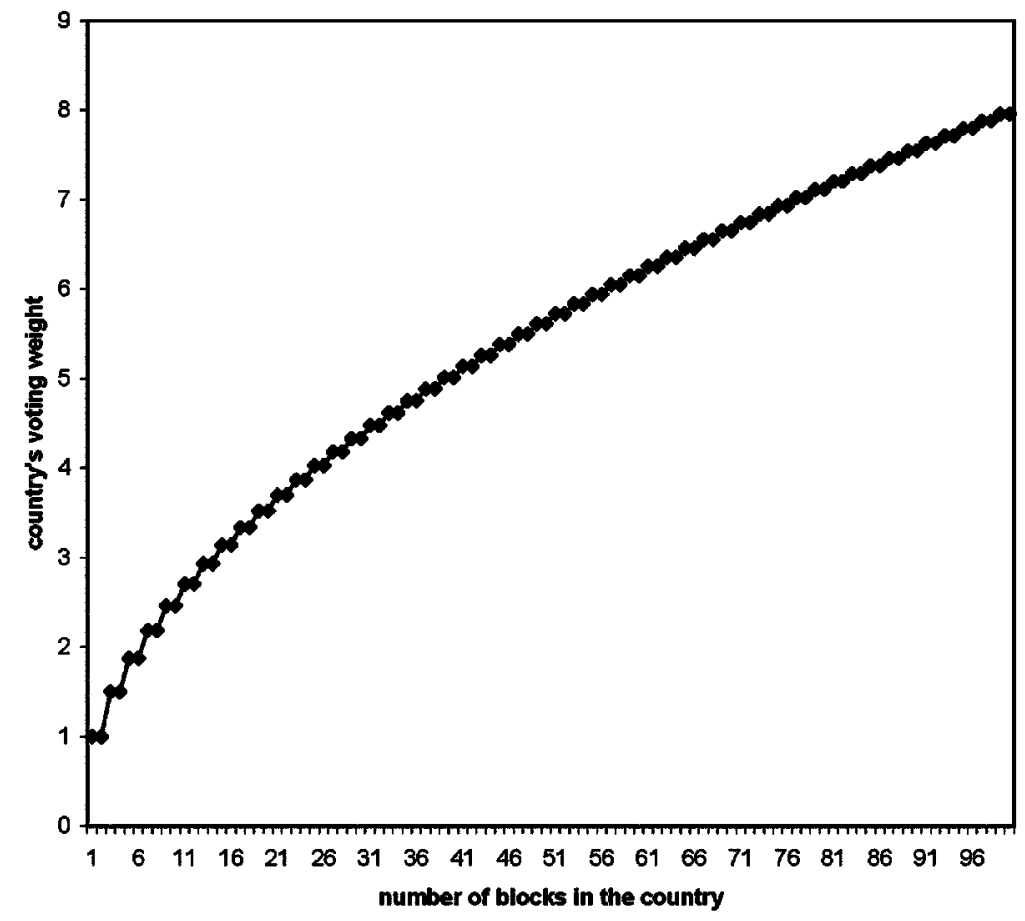

FIG. 1

\section{B. Efficient Weights in the Fixed-Size-Block Model}

Given that the population size of a block $\left(p_{i}\right)$ is the same across all countries, they can be canceled out, and the weights in the fixed-sizeblock-model reduce to

$$
w_{i}^{F S}=2^{-N_{i}} \sum_{x>N_{i} / 2}\left(2 x-N_{i}\right) \frac{N_{i} !}{x !\left(N_{i}-x\right) !} .
$$

These weights are graphed in figure 1 as a function of the number of blocks in the country. ${ }^{14}$

For large numbers of blocks, the weights vary with the square root of the number of blocks, which is consistent with weights originally proposed by Penrose (1946); for small numbers of blocks the weights diverge from this.

${ }^{14}$ Note that the weights are the same for one and two blocks, three and four blocks, etc. This is reflective of the expression in (2). 


\section{Efficient Weights in the Fixed-Number-of-Blocks Model}

In the fixed-number-of-blocks model, as the number of blocks $\left(N_{i}\right)$ is the same in all countries, the difference in the weights then comes only in how many agents are represented in a block. The weights are equivalently directly proportional to the population size of the countries:

$$
w_{i}^{F N}=p_{i}
$$

\section{Asymmetries and Nonmonotonicities in Expected Utilities}

Our perspective has been to maximize the sum of expected utilities, which is quite different from trying to equalize expected utilities across agents. We now illustrate this difference explicitly in the context of the block models. Efficient rules necessarily treat agents asymmetrically, depending on the size of the country they live in. In the following proposition, we compare the expected utilities of agents living in two countries of different population size, under the efficient voting rule in the two variations of the block model.

Proposition 1. In the fixed-number-of-blocks model, agents living in a larger country have expected utilities that are at least as large as those of agents living in a smaller country; and whenever the two countries' weights are not equivalent, ${ }^{15}$ the agents in the larger country have a strictly higher expected utility. In the fixed-size-block model, the expected utilities of agents across countries can be ordered in either direction relative to the ordering of the countries' population sizes.

The proof of the proposition is straightforward. We offer a simple argument for the fixed-number-of-blocks model and an example showing the ambiguity for the fixed-size-block model. In the fixed-numberof-blocks model, any agent's block in any country has exactly the same probability of agreeing with the agent's representative's vote. Thus the expected utilities of agents in different countries differ only to the extent that their representatives receive different weights. As larger countries have larger weights, the claim in the proposition follows directly. ${ }^{16}$

To see the ambiguity in the fixed-size-block model, consider a union

\footnotetext{
${ }^{15}$ Two countries' weights are equivalent if there exists a set of weights that lead in an equivalent voting rule in which the two countries' weights are identical.

${ }^{16}$ As pointed out by a referee, in the extreme case in which countries are actually formed as fairly homogeneous entities (as suggested, e.g., by Alesina and Spolaore [1997]), we would be in a situation in which each country was a single block. This would convey the maximal information possible from a representative's vote, and the overall voting rule would end up being completely efficient.
} 
TABLE 1

Nonmonotonicities in Expected Utilities

\begin{tabular}{lccc}
\hline \hline $\begin{array}{l}\text { Populations of } \\
\text { Countries in } \\
\text { Blocks }\end{array}$ & $\begin{array}{c}\text { Efficient Voting } \\
\text { Weights }\end{array}$ & \multicolumn{2}{c}{$\begin{array}{c}\text { Expected Utility of AN } \\
\text { Agent IN }\end{array}$} \\
\cline { 2 - 4 }$(1,1,1)$ & $(1,1,1)$ & .25 & .25 \\
$(1,1,3)$ & $(1,1,1.5) \sim(1,1,1)$ & .25 & .125 \\
$(1,1,5)$ & $(1,1,1.875) \sim(1,1,1)$ & .25 & .09375 \\
$(1,1,7)$ & $(1,1,2.186) \sim(0,0,1)$ & .15625 \\
$(2,2,7)$ & $(1,1,2.186) \sim(0,0,1)$ & .15625 \\
$(3,3,7)$ & $(1.5,1.5,2.186) \sim(1,1,1)$ & .125 & .078125 \\
\hline
\end{tabular}

of three countries. Table 1 shows the expected utilities of the agents as we vary the number of blocks in the various countries. ${ }^{17}$

The changes in voting weights result in nonmonotonicities in expected utilities in several ways. In the cases of $(1,1,3)$ and $(1,1,5)$, an agent in country 1 or 2 has a higher utility than an agent in country 3 . However, once country 3 hits a population of seven, its weight is such that the votes from countries 1 and 2 are irrelevant. Thus an agent would rather be in the larger country when the configuration is $(1,1$, 7 ), and an agent would prefer to be in a smaller country when the configuration is $(1,1,3)$ or $(1,1,5)$. Also, as we increase country 3 's population from three to five, its agents' utilities fall; but then increasing the population from five to seven leads to an increase in its agents' utilities. This contrasts with decreases in utilities of agents in the other countries.

This example shows that there are no regularities concerning agents' utilities in the fixed-size-block model. The difficulty is that changes in population can dilute a given agent's impact within a country but can also lead to a relative increase of that country's voting weight. As these two factors move against each other, changes can lead to varying effects.

Another question is, how does the total expected utility vary under efficient voting rules as we change the division of a given population into different districts or countries? This issue is also generally ambiguous, regardless of which version of the block model one considers. For instance, one might conjecture that if we start with one division of a

\footnotetext{
${ }^{17}$ If an agent prefers $a$, then he or she gets a payoff of one when his or her preferred outcome is chosen and zero otherwise. If an agent prefers $b$, then he or she gets a payoff of zero when $b$ is chosen and minus one if $a$ is chosen. Then, e.g., for an agent in country 1 in the $(1,1,1),(1,1,3)$, and $(1,1,5)$ cases, there is a three-fourths chance that at least one of the other countries will prefer the agent's preferred alternative and a one-fourth chance that the other two countries will both favor the other alternative. So, in the one-half probability case in which the agent prefers $a$, his or her expected utility is $\frac{3}{4}(1)+\frac{1}{4}(0)$, and in the one-half probability case in which the agent prefers $b$, it is $\frac{3}{4}(0)+\frac{1}{4}(-1)$. Overall this sums to .25 . The calculations in each other case are similarly direct.
} 
population into districts and then further subdivide the population into finer districts, we would enhance efficiency since agents would become closer to their representatives. However, this is not always the case. To see this, note that with a union of just one district or country, we essentially have direct democracy. This is the most efficient case possible. But then dividing this into several districts or countries would lead to a lower total expected utility under the efficient rule than having just one district. Now, if we continue to further subdivide the districts, we eventually reach a point at which each agent resides in a district of one, which brings us back to direct democracy and full efficiency! Generally, subdivisions lead to conflicting changes: on the one hand, having a smaller number of agents within a district gives them a better say in the determination of their representative's vote; on the other hand, their representative is now just one among many. This leads to nonmonotonicities and ambiguities of the types discussed above. ${ }^{18}$

\section{The European Union}

We now examine the voting rules of the Council of Ministers of the European Union under the Nice Treaty and under the draft of the Constitution produced by the Constitutional Convention in June 2003 (Article 24). Given the stylized nature of the block model and the fact that the overall decision-making process of the European Union goes far beyond votes by the Council of Ministers, this is more of an exploratory exercise than a hard commentary on the E.U. voting process. This is also not a positive exercise, but rather a normative one. Our analysis provides a normative description of how voting systems should be designed. In examining the various proposals for E.U. weights, we are not presuming that they are optimal systems. Instead, we discuss which variation of the block model would justify a given proposal.

The voting rule for the European Council of Ministers under the Nice Treaty is weighted voting. At least 255 of the 345 weighted votes (73.9 percent) must be cast in approval of a proposal for it to pass. ${ }^{19}$ The

${ }^{18}$ This leads to a basic trade-off in structuring an indirect democracy. There are tradeoffs between the cost of involving more voters and having more precise representation. There has been little exploration of such trade-offs, either theoretically or empirically, and they might help explain why one sees attempts at more centralization in some cases (e.g., the European Union) and yet more decentralization within some states.

${ }^{19}$ There are two other qualifications as well: (i) that the votes represent at least 14 of the 27 countries and (ii) that the votes represent at least 62 percent of the total population. Calculations by Bräuninger and König (2001) suggest that there are relatively few scenarios in which the weighted vote threshold of 255 votes would be met but one of the other two criteria would fail. It appears that the only impact will arise from the population threshold and that this will involve only a few configurations of votes providing a very slight boost in power to Germany and a slight decrease in power to Malta. Thus, for practical purposes, these additional considerations are relatively unimportant, and the voting weights them- 
relative voting weights appear in figure 2 . We also examine the efficient voting under the two block models. The efficient weights in the fixedsize-block model are calculated for a block size of 1 million. So, for instance, Germany has 83 blocks, France has 59, Italy has 58, and so forth. This leads to efficient voting weights of 7.3, 6.2, and 6.1, respectively, for these countries. The efficient weights in the fixed-number-ofblocks model are simply proportional to population. These also directly correspond to those proposed under the Constitution. In order to make comparisons, we rescale the weights so that they sum to one.

The relationship between the four different weighted voting rules is pictured in figure 2.

A regression of the Nice Treaty weights on the efficient weights under the fixed-size-block model provides an $R^{2}$ of 96 percent for the case of blocks of 1 million (and 95 percent for the case of blocks of 2 million, with $F$-statistics in each case over 600). As a comparison, the fit using weights proportional to population is only 81 percent (with an $F$-statistic of 102), and so the efficient weights under the fixed-size-block model provide a much closer match to the Nice Treaty weights. The reverse is true for the proposed voting rule in the Constitution, with weights that are proportional to population. That rule would not be very efficient if the world were well approximated by the fixed-size-block model, but would be a perfect fit under the fixed-number-of-blocks model.

Thus we are left with an empirical issue. If the world is a good match to the fixed-size-block model, then the Nice Treaty weights are (approximately) efficient; if the world is a good match to the fixed-numberof-blocks model, then the new Constitution's weights are efficient. Of course, these are highly stylized models, and it is likely that the world does not conform to either. While it seems clear that countries such as Luxembourg and Malta consist of more than one block, it also seems clear that the smallest countries have fewer voting blocks than the largest ones. This suggests that the weights should be nonlinear, although perhaps not quite to the level suggested by the fixed-size-block model. A detailed empirical investigation of voting patterns within the countries of the European Union is beyond the scope of this article. ${ }^{20}$

Let us also comment briefly on the voting thresholds. The threshold under the Nice Treaty is 73.9 percent of the total weight, which would be efficient if countries had a bias of roughly $\gamma=3$. This indicates a strong bias for the status quo. In contrast, the threshold of 65 percent under the Constitution would be efficient if countries had a bias of

selves are the main component of the voting procedure. There are discrepancies in the Nice Treaty in that some statements imply a threshold of 258 votes and others a threshold of 255 votes. It appears that the correct number is 255 .

${ }^{20}$ See Barberà and Jackson (2004b) for a preliminary analysis of estimated optimal voting weights based on poll data from the Eurobarometer $(2003 a, 2003 b)$. 


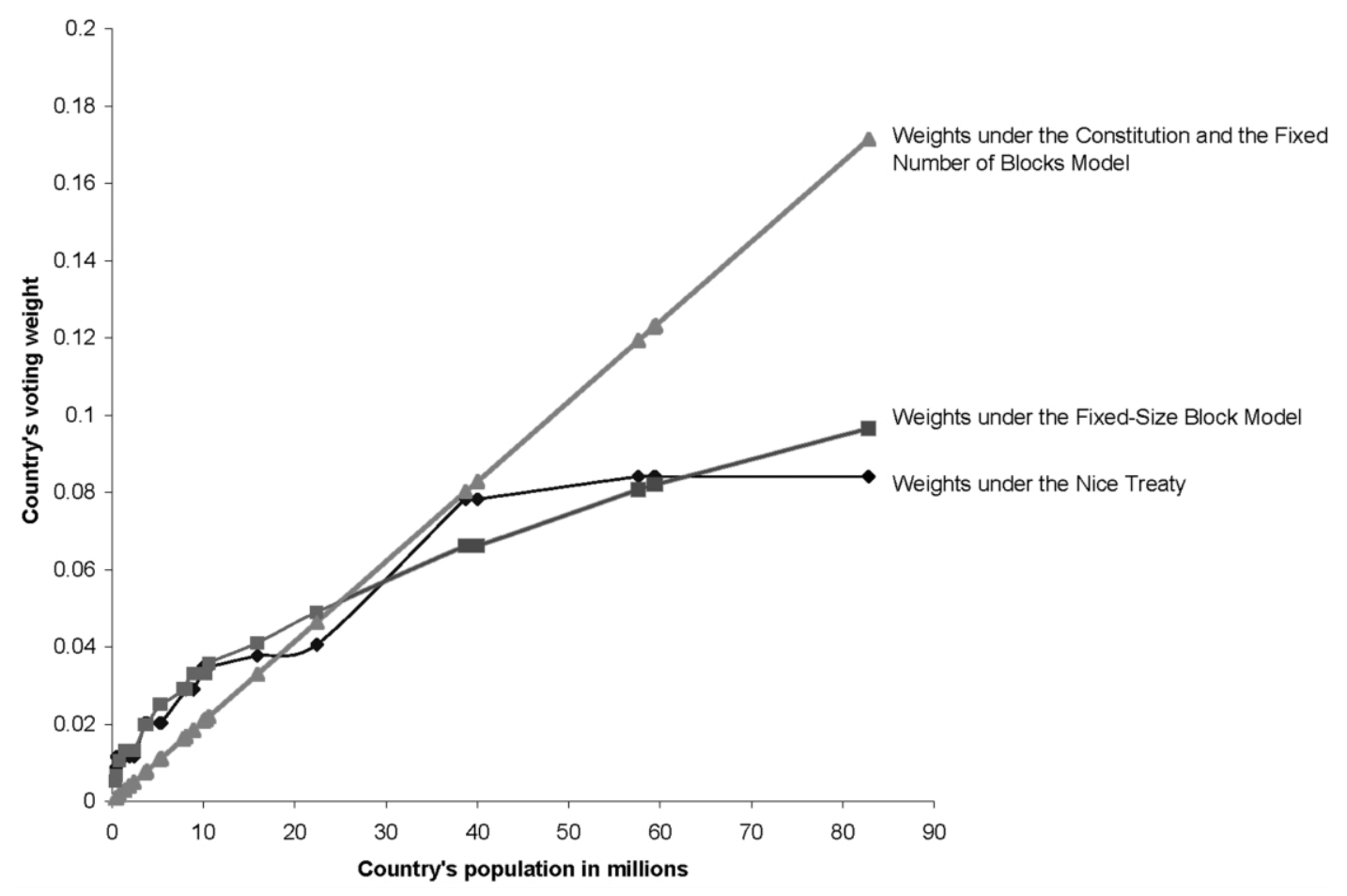

FIG. 2 
roughly $\gamma=1.86$. This is also a bias for the status quo, but a less pronounced one.

\section{Concluding Remarks}

We have provided a framework for designing and analyzing efficient voting rules in the context of indirect democracy. We have shown that the model can be applied to analyzing voting rules such as those of the European Union and that the relative merits of different rules reduce to readily identifiable hypotheses that are amenable to empirical testing. A careful analysis of the E.U. voting rules will require richer data and application of our results beyond the case of the block model. Nevertheless, our theoretical results provide a framework with intuitive characterizations of optimal voting rules that appears to lend itself well to such an exercise.

There are other considerations that should be explored in further studies. In decision making, it may be that countries can sometimes include side payments or logroll so that multiple decisions can be made at once. These possibilities can further enhance the efficiency of the decision making and might influence the structure of the voting system that is to be used. ${ }^{21}$ One can also consider a voting system's stability. As the rules can be amended, considerations other than efficiency enter the long-run picture, since only certain rules will survive..$^{22}$ Another is the issue of fairness or equality. As we have shown, efficient weights do not necessarily lead to the same expected utilities for agents in different countries. For instance, proposition 1 showed that larger countries are favored under proportional weights in the fixed-number-of-blocks model. There are other issues that can be considered, such as votes over more than two alternatives, private information, agenda formation, and risk aversion, to highlight a few of the more obvious ones.

Appendix

\section{Proof of Theorem 1}

An efficient voting rule is a feasible voting rule that maximizes

$$
E\left[\sum_{k} v(\boldsymbol{u}) u_{k}\right] .
$$

\footnotetext{
${ }^{21}$ See, e.g., Harstad (2004, 2005), who considers side payments and the ability to invest in projects; Casella (2005), who considers rules specifically designed for votes over sequences of decisions; and Jackson and Sonnenschein (forthcoming), who show that approximate efficiency can be obtained if multiple decisions can be bundled and voted over in a linked manner.

${ }^{22}$ See Barberà and Jackson (2004a) and Sosnowska (2002) for an examination of the stability of voting rules.
} 
Let $\left(r_{1}(\boldsymbol{u}), \ldots, r_{m}(\boldsymbol{u})=r_{1}, \ldots, r_{m}\right)$ be an event in which the realization of representatives (i.e., votes of the countries) is $\left(r_{1}, \ldots, r_{m}\right) \in\{a, b\}^{m}$. Under feasibility, we can then write $v(\boldsymbol{u})$ as a function of $\left(r_{1}, \ldots, r_{m}\right)$ instead of $\boldsymbol{u}$. Hence, the total expected utility is written as

$$
\begin{gathered}
\sum_{r_{1}, \ldots, r_{m}} E\left[\sum_{k} v\left(r_{1}, \ldots, r_{m}\right) u_{k} \mid r_{1}(\boldsymbol{u}), \ldots, r_{m}(\boldsymbol{u})=r_{1}, \ldots, r_{m}\right] \\
\times P\left(r_{1}(\boldsymbol{u}), \ldots, r_{m}(\boldsymbol{u})=r_{1}, \ldots, r_{m}\right) .
\end{gathered}
$$

Given the independence across countries, we can write this as

$$
\sum_{r_{1}, \ldots, r_{m}} v\left(r_{1}, \ldots, r_{m}\right)\left\{\sum_{i} E\left[\sum_{k \in C_{i}} u_{k} \mid r_{i}(\boldsymbol{u})=r_{i}\right]\right\} P\left(r_{1}(\boldsymbol{u}), \ldots, r_{m}(\boldsymbol{u})=r_{1}, \ldots, r_{m}\right) .
$$

It then follows that if we can find a voting rule that maximizes

$$
v\left(r_{1}, \ldots, r_{m}\right) \sum_{i} E\left[\sum_{k \in C_{i}} u_{k} \mid r_{i}\right]
$$

pointwise for each $\left(r_{1}, \ldots, r_{m}\right)$, then it must be efficient. Moreover, if we find one that leads to a zero whenever there is indifference between $a$ and $b$, then all efficient voting rules must be equivalent to it up to ties.

Note that for any given $\left(r_{1}, \ldots, r_{m}\right)$, maximizing expression (A1) requires setting $v\left(r_{1}, \ldots, r_{m}\right)=1$ when

$$
\sum_{i} E\left[\sum_{k \in C_{i}} u_{k} \mid r_{i}\right]>0
$$

and $v\left(r_{1}, \ldots, r_{m}\right)=0$ when

$$
\sum_{i} E\left[\sum_{k \in C_{i}} u_{k} \mid r_{i}\right]<0
$$

and does not have any requirement when this expression is equal to zero.

Given the definitions of $w_{i}^{a}$ and $w_{i}^{b}$, we can then rewrite (A2) and (A3) as $v\left(r_{1}, \ldots, r_{m}\right)=1$ when

$$
\sum_{i: r_{i}=a} w_{i}^{a}-\sum_{i: r_{i}=b} w_{i}^{b}>0
$$

and $v\left(r_{1}, \ldots, r_{m}\right)=0$ when

$$
\sum_{i: r_{i}=a} w_{i}^{a}-\sum_{i: r_{i}=b} w_{i}^{b}<0
$$

This is as defined in $v^{E}$, where we flip a coin in the case of a tie. Any efficient voting rule must agree with this one except in the case in which this rule results in an expression equal to zero. This concludes the proof of the theorem. QED

\section{References}

Aghion, Philippe, and Patrick Bolton. 2003. "Incomplete Social Contracts.” J. European Econ. Assoc. 1 (March): 38-67. 
Alesina, Alberto, and Enrico Spolaore. 1997. "On the Number and Size of Nations." Q.J.E. 112 (November): 1027-56.

Badger, Wade W. 1972. "Political Individualism, Positional Preferences, and Optimal Decision-Rules." In Probability Models of Collective Decision Making, edited by Richard G. Niemi and Herbert F. Weisberg. Columbus, OH: Merrill.

Baldwin, Richard E., Erik Berglöf, Francesco Giavazzi, and Mika Widgrén. 2001. Nice Try: Should the Treaty of Nice Be Ratified? London: Centre Econ. Policy Res.

Banzhaf, John F., III. 1965. "Weighted Voting Doesn't Work: A Mathematical Analysis." Rutgers Law Rev. 19 (Winter): 317-43.

Barberà, Salvador, and Matthew O. Jackson. 2004a. "Choosing How to Choose: Self-Stable Majority Rules and Constitutions.” Q.J.E. 119 (August): 1011-48.

. 2004b. "On the Weights of Nations: Assigning Voting Weights in a Heterogeneous Union.” Social Sciences Working Paper no. 1196, California Inst. Tech.

Barry, Brian. 1980. "Is It Better to Be Powerful or Lucky?” Pts. 1 and 2. Political Studies 28 (2): 183-94; 28 (3): 338-52.

Beisbart, Claus, Luc Bovens, and Stephan Hartmann. 2005. "A Utilitarian Assessment of Alternative Decision Rules in the Council of Ministers." European Union Politics 6 (4): 395-418.

Bovens, Luc, and Stephan Hartmann. 2004. "Welfare, Voting and the Constitution of a Federal Assembly." Manuscript, London School Econ.

Bräuninger, Thomas, and Thomas König. 2001. "Voting Power in the Post-Nice European Union.” Manuscript, Univ. Konstanz.

Casella, Alessandra. 2005. "Storable Votes." Games and Econ. Behavior 51 (May): 391-419.

Curtis, Richard B. 1972. "Decision Rules and Collective Values in Constitutional Choice." In Probability Models of Collective Decision Making, edited by Richard G. Niemi and Herbert F. Weisberg. Columbus, OH: Merrill.

Dubey, Pradeep, and Lloyd S. Shapley. 1979. "Mathematical Properties of the Banzhaf Power Index." Math. Operations Res. 4 (May): 99-131.

Eurobarometer. 2003a. "Eurobarometer 60: Public Opinion in the European Union.” European Union Research Group, Brussels. http://europa.eu.int/ comm/public_opinion/archives/eb/eb60/eb60_en.pdf.

. 2003b. "Eurobarometer 2003b. "Public Opinion in the Acceding and Candidate Countries.” European Union Research Group, Brussels. http:// europa.eu.int/comm/public_opinion/archives/cceb/2003/cceb2003.4_first _annexes.pdf.

Feix, Marc R., Dominique Lepelley, Vincent Merlin, and Jean-Louis Rouet. 2004. "Fair and Efficient Representation of the Citizens in a Federal Union." Manuscript, SUBATECH, Nantes.

Felsenthal, Dan S., and Moshé Machover. 1999. "Minimizing the Mean Majority Deficit: The Second Square-Root Rule.” Math. Soc. Sci. 37 (January 1): 25-37.

Galloway, David. 2001. The Treaty of Nice and Beyond: Realities and Illusions of Power in the EU. Sheffield, U.K.: Sheffield Academic Press.

Harstad, Bård. 2004. "Harmonization and Side Payments in Political Cooperation." Manuscript, Northwestern Univ.

. 2005. "Majority Rules and Incentives." Q.J.E. 120 (November): 1535-68.

Jackson, Matthew O., and Hugo F. Sonnenschein. Forthcoming. "Overcoming Incentive Constraints by Linking Decisions." Econometrica.

Laruelle, Annick. 1998. "Game Theoretical Analysis of Decision-Making Processes with Applications to the European Union.” PhD diss., Univ. Catholique de Louvain. 
Laruelle, Annick, and Federico Valenciano. 2003. "Assessing Success and Decisiveness in Voting Situations.” Manuscript, Univ. Basque Country.

Laruelle, Annick, and Mika Widgrén. 1998. "Is the Allocation of Voting Power among EU States Fair?" Public Choice 94 (3/4): 317-39.

Leech, Dennis. 2002. "Designing the Voting System for the Council of the European Union.” Public Choice 113 (December): 437-64.

Penrose, L. S. 1946. "The Elementary Statistics of Majority Voting." J. Royal Statis. Soc. 109 (1): 53-57.

Rae, Douglas W. 1969. "Decision-Rules and Individual Values in Constitutional Choice." American Polit. Sci. Rev. 63 (March): 40-56.

Shapley, Lloyd S., and Martin Shubik. 1954. "A Method for Evaluating the Distribution of Power in a Committee System.” American Polit. Sci. Rev. 48 (September): 787-92.

Sosnowska, Honorata. 2002. "A Generalization of the Barbera-Jackson Model of Self-Stable Majority Rules." Manuscript, Warsaw School Econ.

Sutter, Matthias. 2000. "Fair Allocation and Re-weighting of Votes and Voting Power in the EU before and after the Next Enlargement." J. Theoretical Politics 12 (4): 433-49. 